Pacific Journal of Mathematic 


\title{
A CHARACTERIZATION OF COMPLETE LATTICES
}

\author{
ANNE C. DAvis
}

1. Introduction. A complete lattice $\mathfrak{U}=\langle A, \leq\rangle$ has the property that every increasing function on $A$ to $A$ has a fixpoint. ${ }^{1}$ Tarski raised the question whether the converse of this result also holds. In this note we shall show that the answer to this question is affirmative, thus establishing a criterion for completeness of a lattice in terms of fixpoints. ${ }^{2}$

We shall use the notation of [6]. In addition, the formula $a \notin b$ will be used to express the fact that $a \leq b$ does not hold. By $\left\langle a_{\xi} ; \xi\langle\alpha\rangle\right.$, where $\alpha$ is any (finite or transfinite) ordinal we shall denote the sequence whose consecutive terms are $a_{0}, a_{1}, \ldots, a_{\xi}, \ldots$ (with $\xi<\alpha$ ); the set of all terms of this sequence will be denoted by $\left\{a_{\xi} ; \xi<\alpha\right\}$. The sequence $\left\langle a_{\xi} ; \xi<\alpha\right\rangle$ is, of course, called increasing, or strictly increasing, if $a_{\xi} \leq a_{\xi^{\prime}}$, or $a_{\xi}<a_{\xi^{\prime}}$, for any $\xi<\xi^{\prime}<\alpha$; analogously we define decreasing and strictly decreasing sequences.

2. A lemma. We start with the following:

LEMma 1. If the lattice $\mathfrak{Y}=\langle A, \leq\rangle$ is incomplete, then there exist two sequences $\left\langle b_{\xi} ; \xi<\beta\right\rangle$ and $\left\langle c_{\eta} ; \eta<\gamma\right\rangle$ such that

(i) $b_{\xi}<c_{\eta}$ for every $\xi<\beta$ and every $\eta<\gamma$,

(ii) $\left\langle b_{\xi} ; \xi\langle\beta\rangle\right.$ is strictly increasing and $\left\langle c_{\eta} ; \eta\langle\gamma\rangle\right.$ is strictly decreasing,

(iii) there is no element $a \in A$ which is both an upper bound of $\left\{b_{\xi} ; \xi<\beta\right\}$ and a lower bound of $\left\{c_{\eta} ; \eta<\gamma\right\}^{3}$

${ }^{1}$ See [6] (where further historical references can also be found).

2 This result was found in 1950 and outlined in [2].

${ }^{3}$ A related, though weaker, property of incomplete lattices is mentioned implicitly in $[1$, p. 53, Exercise 4$]$.

Received June 29, 1953. The present note was prepared while the author was working on a research project in the foundations of mathematics sponsored by the Office of Ordnance Research, U.S. Army.

Pacific J. Math. 5 (1955), $311-319$ 
Proof. We first notice that there exists at least one subset of $A$ without a least upper bound (for otherwise the lattice would be complete). ${ }^{4}$ Hence we can find a subset $B$ of $A$ with the following properties:

$$
\text { UB does not exist; }
$$

(2) if $X$ is any subset of $A$ with smaller power than $B$, then $\cup X$ exists.

Let $\beta^{\prime}$ be the initial ordinal of the same power as $B$ (that is, the smallest ordinal such that the set of all preceding ordinals has the same power as $B$ ). The ordinal $\beta^{\prime}$ may be equal to 0 ; if not, $\beta^{\prime}$ is certainly infinite and, since it is initial, it has no predecessor; that is, $\xi<\beta^{\prime}$ implies $\xi+1<\beta^{\prime}$ for every ordinal $\xi$. Thus all the elements of $B$ can be arranged in a sequence $\left\langle b_{\xi}^{\prime}\right.$; $\left.\xi<\beta^{\prime}\right\rangle$. For every $\xi<\beta^{\prime}$, the set $\left\{b_{\zeta}^{\prime} ; \zeta<\xi+1\right\}$ is of smaller power than $\beta^{\prime}$ and therefore, by (2), its least upper bound

$$
u_{\xi}=U\left\{b_{\zeta}^{\prime} ; \zeta<\xi+1\right\}
$$

exists. The sequence $\left\langle u_{\xi} ; \xi<\beta^{\prime}\right\rangle$ is clearly increasing but not necessarily strictly increasing. However, by omitting repeating terms in this sequence, we obtain a strictly increasing sequence $\left\langle b_{\xi} ; \xi<\beta\right\rangle$, where $\beta$ is an ordinal $\leq \beta^{\prime}$, such that

$$
\left\{b_{\xi} ; \xi<\beta\right\}=\left\{u_{\xi} ; \xi<\beta^{\prime}\right\}
$$

(Actually, one can easily prove that $\beta=\beta^{\prime}$.) Obviously,

$$
\text { for every } b \in B \text { there is a } \xi<\beta \text { such that } b \leq b_{\xi} \text {; }
$$$$
\text { for every } \xi<\beta^{\prime} \text { there is a subset } X \text { of } B \text { such that } b_{\xi}=U X \text {. }
$$

By (3) and (4), if the least upper bound $U\left\{b_{\xi} ; \xi<\beta\right\}$ existed, it would coincide with $\cup B$; hence, by (1),

$$
\cup\left\{b_{\xi} ; \xi<\beta\right\} \text { does not exist. }
$$

Let $C$ be the set of all upper bounds of $\left\{b_{\xi} ; \xi<\beta\right\}$. Clearly $\cap C$ does not exist, for if it did, it would coincide with $U\left\{b_{\xi} ; \xi<\beta\right\}$; this result would contradict (5). Now $C$, like $B$, is either empty or infinite. Since $C$ is partly ordered by the relation $\leq$, there is a strictly decreasing sequence $\left\langle c_{\eta} ; \eta\langle\gamma\rangle\right.$ such 
that $\left\{c_{\eta} ; \eta<\gamma\right\}$ is a subset of $C$ with which $C$ is coinitial (that is, there is no element of $C$ which is a lower bound of $\left\{c_{\eta} ; \eta<\gamma\right\}$ without belonging to $\left.\left\{c_{\eta} ; \eta<\gamma\right\}\right) .{ }^{5}$ If the greatest lower bound $\cap\left\{c_{\eta} ; \eta<\gamma\right\}$ existed, it would be an upper bound of $\left\{b_{\xi} ; \xi<\beta\right\}$; but since $U\left\{b_{\xi} ; \xi<\beta\right\}$ does not exist, there would be an element $c \in C$ such that $\cap\left\{c_{\eta} ; \eta<\gamma\right\} \nsubseteq c$. Hence we would have

$$
c \cap \cap\left\{c_{\eta} ; \eta<\gamma\right\} \in C \text { and } c \cap \cap\left\{c_{\eta} ; \eta<y\right\}<\cap\left\{c_{\eta} ; \eta<\gamma\right\} \text {, }
$$

in contradiction to the assumption that $C$ is coinitial with $\left\{c_{\eta} ; \eta<\gamma\right\}$. Consequently,

$$
\cap\left\{c_{\eta} ; \eta<y\right\} \text { does not exist. }
$$

The sequences $\left\langle b_{\xi} ; \xi<\beta\right\rangle$ and $\left\langle c_{\eta} ; \eta<\gamma\right\rangle$ obviously satisfy conditions (i) and (ii) of our lemma. To show that (iii) is also satisfied, assume that an element $a \in A$ is both an upper bound of $\left\{b_{\xi} ; \xi<\beta\right\}$ and a lower bound of $\left\{c_{\eta} ; \eta<\gamma\right\}$. We have then, by definition, $a \in C$. Hence, $C$ being coinitial with $\left\{c_{\eta} ; \eta<y\right\}$, we must have

$$
a \in\left\{c_{\eta} ; \eta<\gamma\right\}
$$

and therefore

$$
a=\cap\left\{c_{\eta} ; \eta<\gamma\right\},
$$

in contradiction to (6). This completes the proof.

3. The main result. With the help of Lemma 1 we now obtain the main result of this note:

THE OREM 2. For a lattice $\mathcal{U}=\langle A, \leq\rangle$ to be complete it is necessary and sufficient that every increasing function on $A$ to $A$ have a fixpoint.

Proof. Since the condition of the theorem is known to be necessary for the completeness of a lattice, we have only to show that it is sufficient. In other words, we have to show that, under the assumption that the lattice $2 \mathbb{U}=\langle A, \leq\rangle$ is incomplete, there exists an increasing function $f$ on $A$ to $A$ without fixpoints.

In fact, let $\left\langle b_{\xi} ; \xi<\beta\right\rangle$ and $\left\langle c_{\eta} ; \eta\langle\gamma\rangle\right.$ be any two sequences satisfying conclusions (i)-(iii) of Lemma 1. To define $f$ for any element $x \in A$, we distinguish two cases dependent upon whether $x$ is a lower bound of $\left\{c_{\eta} ; \eta<\gamma\right\}$ or not. 
In the first case, by conclusion (iii), $x$ is not an upper bound of $\left\{b_{\xi} ; \xi<\beta\right\}$; that is, the set of ordinals

$$
\Phi(x)=E_{\xi}\left[\xi<\beta \text { and } b_{\xi} \Varangle x\right]
$$

is non-empty. We put

$$
\phi(x)=\min \Phi(x) \text { and } f(x)=b_{\phi(x)} \cdot
$$

( $\Delta$ being any non-empty set of ordinals, min $\Delta$ is of course the smallest ordinal belonging to $\Delta$.) In the second case, the set

$$
\Psi(x)=E_{\eta}\left[\eta<\gamma \text { and } x \notin c_{\eta}\right]
$$

is nonempty. We let

$$
\psi(x)=\min \Psi(x) \text { and } f(x)=c_{\psi(x)}
$$

We have thus defined a function $f$ on $A$ to $A$. From (1)-(4) it follows clearly that either $f(x) \$ x$ or $x \notin f(x)$ for every $x \in A$; thus $f$ has no fixpoints.

Let $x$ and $y$ be any elements of $A$ with $x \leq y$. If $x$ is a lower bound of $\left\{c_{\eta} ; \eta<\beta\right\}$ but $y$ is not, then, by (1)-(4) and conclusion (i) of Lenma 1, $f(x) \leq f(y)$. If both $x$ and $y$ are lower bounds of $\left\{c_{\eta}, \eta<\gamma\right\}$, we see from (1) that $\Phi(y)$ is a subset of $\Phi(x)$; hence, by (2) and conclusion (ii) of Lemma 1 , it follows at once that $f(x) \leq f(y)$. Finally, if $x$ is not a lower bound of $\left\{c_{\eta} ; \eta<\gamma\right\}$, then $y$ is not either, and by an argument analogous to that just outlined (using (3) and (4) instead of (1) and (2)) we again obtain $f(x) \leq f(y)$. Thus the function $f$ is increasing, and the proof of the theorem is complete.

4. Extensions. More difficult problems seem to arise if we try to improve Theorem 2 by considering, instead of arbitrary increasing functions, more special classes of functions. In particular, we have in mind join-distributive (or meet-distributive) functions, that is, functions $f$ on $A$ to $A$ which satisfy the formula

$$
f(x \cup y)=f(x) \cup f(y)(\text { or } f(x \cap y)=f(x) \cap f(y))
$$

for all $x, y \in A$. The problem is open whether Theorem 2 remains valid if the term "increasing" is replaced by "join-distributive" or by "meet-distributive". We are going to give (in Theorem 4 below) a partial positive result concerning this problem. 
The lattice $\mathcal{Z U}=\langle A, \leq\rangle$ is called $\alpha$-join-complete (or $\alpha$-meet-complete $)$ if $\mathrm{U} X($ or $\cap X)$ exists for every nonempty subset $X$ of $A$ with power at most equal to $\boldsymbol{s}_{\alpha}$.

Lemma 3. Let $\mathfrak{U}=\langle A, \leq\rangle$ be an incomplete lattice with the set $A$ of power

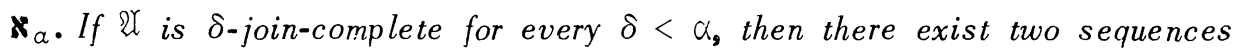
$\left\langle b_{\xi} ; \xi<\beta\right\rangle$ and $\left\langle c_{\eta} ; \eta<\gamma\right\rangle$ which satisfy conclusions (i)-( iii) of Lemma 1 as well as the following condition:

(iv) if an element $x \in A$ is a lower bound of $\left\{c_{\eta} ; \eta<\gamma\right\}$, then there exists an ordinal $\xi$ such that $\xi<\beta$ and $x \leq b_{\xi}$.

Proof. From Lemma 1 we easily conclude that there exists a strictly decreasing sequence $\left\langle c_{\eta} ; \eta<\gamma\right\rangle$ of elements of $A$ such that $\cap\left\{c_{\eta} ; \eta<\gamma\right\}$ does not exist. Let $B^{\prime}$ be the set of all lower bounds of $\left\{c_{\eta} ; \eta<\gamma\right\}$. Then clearly $\cup B^{\prime}$ does not exist. Hence, by hypothesis, $B^{\prime}$ must be either empty or of power $\boldsymbol{\kappa}_{\alpha}$; since $\mathfrak{A}$ is $\delta$-join-complete for every $\delta<\alpha$, it follows that $B^{\prime}$ satisfies conditions ( 1 ) and (2) in the proof of Lemma 1 (with $B$ replaced by $B^{\prime}$ ). Therefore, by literally repeating the corresponding part of the proof of that lemma, we obtain a strictly increasing sequence $\left\langle b_{\xi} ; \xi<\beta\right\rangle$ of elements of $B^{\prime}$ for which conditions (3)-(5) (with $B=B^{\prime}$ ) hold. Obviously the sequences $\left\langle b_{\xi} ; \xi<\beta\right\rangle$ and $\left\langle c_{\eta} ; \eta\langle\gamma\rangle\right.$ satisfy conclusions (i) and (ii) of Lemma l. To show that conclusion (iii) is satisfied, assume, to the contrary, that $a$ is both a lower bound of $\left\{c_{\eta} ; \eta<\gamma\right\}$ and an upper bound of $\left\{b_{\xi} ; \xi<\beta\right\}$. Therefore, by the definition of $B^{\prime}$, we have $a \in B^{\prime}$; using (3) of the proof of Lemma 1 we see that $a \leq b_{\xi}$ for some $\xi<\beta$, and hence, $a$ being an upper bound of $\left\{b_{\xi} ; \xi<\beta\right\}$, we conclude that

$$
a=\cup\left\{b_{\xi} ; \xi<\beta\right\},
$$

which contradicts (5). Finally, in view of the definition of $B^{\prime}$, conclusion (iv) of our present lemma simply coincides with condition (3) in the proof of Lemma 1 (again with $B=B^{\prime}$ ).

With the help of Lemma 3 we now obtain:

THEOREM 4. Let $\mathcal{A}=\langle A, \leq\rangle$ be a lattice with the set $A$ of power $\boldsymbol{\aleph}_{a} \cdot$ For $A$ to be complete it is necessary and sufficient that

(i) $\mathfrak{U}$ be $\delta$-join-complete for every $\delta<\alpha$ and

(ii) every join-distributive function on A to A have a fixpoint. 
Proof. If $\mathscr{Y}$ is complete, then obviously (i) holds. To show that the completeness of \& implies ( $i i)$ we need only note that every join-distributive function is increasing, and then apply Theorem 2. Thus (i) and (ii) are necessary conditions for the completeness of $\mathfrak{X}$.

In order to show that these conditions (jointly) are also sufficient, we assume that $\mathscr{O}$ is an incomplete lattice which is $\delta$-join-complete for every $\delta<\alpha$, and we show that there exists a join-distributive function $f$ on $A$ to $A$ without fixpoints.

Let $\left\langle b_{\xi} ; \xi<\beta\right\rangle$ and $\left\langle c_{\eta} ; \eta<\gamma\right\rangle$ be any two sequences satisfying conclusions (i) - (iii) of Lemma 1 and the additional conclusion (iv) of Lemma 3. In order to define $f$ for every $x \in A$ we distinguish two cases dependent upon whether $x$ is a lower bound of $\left\{c_{\eta} ; \eta<\gamma\right\}$ or not.

In the first case, by (iv) of Lemma 3 , the set

$$
\theta(x)=E_{\xi}\left[\xi<\beta \text { and } x \leq b_{\xi}\right]
$$

is non-empty. We notice that, by conclusions (ii) and (iii), the sequence $\left\langle b_{\xi} ; \xi<\beta\right\rangle$ cannot have a last term; that is $\xi<\beta$ always implies $\xi+1<\beta$. Hence we may put

$$
\vartheta(x)=\min \theta(x) \text { and } f(x)=b \vartheta(x)+1 .
$$

In the second case, the set

$$
\Psi(x)=\mathrm{E}_{\eta}\left[\eta<\gamma \text { and } x \notin c_{\eta}\right]
$$

is nonempty. We let

$$
\psi(x)=\min \Psi(x) \text { and } f(x)=c_{\psi(x)} \cdot
$$

We have thus defined a function $f$ on $A$ to $A$. If $x \in A$, and $x$ is a lower bound of $\left\{c_{\eta} ; \eta<\gamma\right\}$, it follows from (1), (2), and conclusion (ii) of Lemma 1, that

$$
x \leq b_{\vartheta(x)}<b_{\vartheta(x)+1}=f(x)
$$

while if $x$ is not a lower bound of $\left\{c_{\eta} ; \eta<\gamma\right\}$, we see from (3) and (4) that $x \notin f(x)$; thus $f$ has no fixpoints.

Now let $x$ and $y$ be any elements of $A$. Assume first that both $x$ and $y$ are lower bounds of $\left\{c_{\eta} ; \eta<\gamma\right\}$. Let, in addition, $\vartheta(x) \leq \vartheta(y)$. Then, obviously,

$$
\vartheta(x)+1 \leq \vartheta(y)+1
$$


and, by (2) and conclusion (ii) of Lemma 1, we obtain

$$
f(x) \cup f(y)=b \vartheta(x)+1 \cup b \vartheta(y)+1=b \vartheta(y)+1 .
$$

Clearly, $x \cup y$ is a lower bound of $\left\{c_{\eta} ; \eta<y\right\}$ and we see from (1) that $\theta(x \cup y)$ is a subset of $\theta(y)$; therefore it follows from (2) that

$$
\vartheta(y) \leq \vartheta(x \cup y) .
$$

On the other hand, by (1), (2), and conclusion (ii) of Lemma 1, we have

$$
x \leq b \vartheta(x) \leq b \vartheta(y) \text { and } y \leq b \vartheta(y)
$$

hence $x \cup y \leq b \vartheta_{(y)}$ and, by (1), $\vartheta(y) \in \theta(x \cup y)$. Then, using (2), we obtain

$$
\vartheta(x \cup y) \leq \vartheta(y)
$$

hence, with the help of (2), (5), and (6), we conclude that

$$
f(x \cup y)=f(x) \cup f(y) .
$$

Assume next that $x$ is a lower bound of $\left\{c_{\eta} ; \eta<\gamma\right\}$ while $y$ is not. Then, by (2), (4), and conclusion (i) of Lemma 1, we have

$$
f(x) \cup f(y)=b \vartheta(x)+1 \cup c_{\psi(y)}=c_{\psi(y)} \cdot
$$

Since $y$ is not a lower bound of $\left\{c_{\eta} ; \eta<\gamma\right\}, x \cup y$ is not either, and by (3) we see that $\Psi(y)$ is a subset of $\Psi(x \cup y)$; therefore, by (4),

$$
\psi(x \cup y) \leq \psi(y) .
$$

From (3) and (4) it is obvious that

$$
x \cup y \pm c_{\psi(x \cup y)},
$$

and hence either

$$
x\rfloor c_{\psi(x \cup y)} \text { or } y \geqq c_{\psi(x \cup y)} \text {. }
$$

But since $x$ is assumed to be a lower bound of $\left\{c_{\eta} ; \eta<\gamma\right\}$, it follows that

$$
y \pm c_{\psi(x \cup y)} ;
$$

therefore, by (3), $\psi(x \cup y) \in \Psi(y)$; and, by (4), 


$$
\psi(y) \leq \psi(x \cup y) .
$$

Applying (4), (8), (9), and (10), we conclude that (7) holds.

Finally, assume that neither $x$ nor $y$ is a lower bound $\left\{c_{\eta} ; \eta<\gamma\right\}$, and let $\psi(x) \leq \psi(y)$. From (4) and conclusion (ii) of Lemma 1, we obtain

$$
f(x) \cup f(y)=c_{\psi(x)} \cup c_{\psi(y)}=c_{\psi(x)} .
$$

Since, by (3), $\Psi(x)$ is a subset of $\Psi(x \cup y)$, it follows from (4) that

$$
\psi(x \cup y) \leq \psi(x) .
$$

Using (3) and (4) again, we see that

$$
x \cup y ₫ c_{\psi(x \cup y)},
$$

and hence either

$$
x \geqq c_{\psi(x \cup y)} \text { or } y \geqq c_{\psi(x \cup y)} \cdot
$$

Therefore,

$$
\psi(x) \leq \psi(x \cup y) \text { or } \psi(y) \leq \psi(x \cup y) .
$$

But if $\psi(y) \leq \psi(x \cup y)$, then, since $\psi(x) \leq \psi(y)$, it is also the case that

$$
\psi(x) \leq \psi(x \cup y) .
$$

Using (4), (11), (12), and (13), we again obtain (7). Thus the function $f$ is join-distributive, and the proof of the theorem is complete.

As an immediate consequence of Theorem 4 we obtain:

Corollary 5. Let $\mathfrak{Q}=\langle A, \leq\rangle$ be a lattice in which the set $A$ is denumerable. For \& to be complete it is necessary and sufficient that every joindistributive function on $A$ to $A$ have a fixpoint.

By analyzing the preceding proofs we easily see that Theorem 4 and Corollary 5 remain valid if we replace in them "join" by "meet" everywhere; we also notice that in every lattice $\mathfrak{U}=\langle A, \leq\rangle$ without 0 the conclusions of Lemma 3 (with $\beta=0$ ) hold, and hence there is a join-distributive function on $A$ to $A$ without fixpoints.

If, instead of considering arbitrary lattices, we restrict ourselves to 
Boolean algebras, we immediately conclude from Corollary 5 that in every Boolean algebra $\mathfrak{U}=\langle A, \leq\rangle$ in which the set $A$ is (infinitely) denumerable there is a join-distributive function $f$ on $A$ to $A$ without fixpoints. This result can be extended to a wider class of Boolean algebras, in fact to all infinite Boolean algebras with an ordered basis; ${ }^{6}$ the proof will not be given here. ${ }^{7}$ However, the question remains open whether the result can be extended to arbitrary incomplete or even to arbitrary countably incomplete Boolean algebras (that is, to those which, in our terminology, are not 0 -join-complete).

\footnotetext{
${ }^{6} \mathrm{~F}$ or the notion of a Boolean algebra with ordered basis, see [5]. It is well known that every denumerable Boolean algebra has an ordered basis, and that every infinite Boolean algebra with an ordered basis is countably incomplete, but that the converses of these statements do not hold.

7 The essential property of infinite Boolean algebras with an ordered basis which is involved in this proof is that every such algebra contains a sequence of disjoint nonzero elements $\left\langle b_{\mu} ; \mu<\omega\right\}$ such that, for every element $x$ of the algebra, either the set $\mathrm{E}_{\mu}\left[b_{\mu} \cap_{x=0]}\right.$ or the set $\mathrm{E}_{\mu}\left[b_{\mu} \cap x \neq 0\right]$ is finite. The idea of the proof was suggested to the author by an argument in $[4, \mathrm{p} .921]$, where a particular case of the result in question was obtained.
}

\section{REFERENCES}

1. Garrett Birkhoff, Lattice theory, revised edition, Amer. Math. Soc. Colloq. Publ. vol. 25, New York, 1948.

2. Anne C. Davis, A fixpoint criterion for completeness of a lattice (preliminary report ), Bull. Amer. Math. Soc. 57 (1951), 77.

3. Felix Hausdorff, Grundzüge der Mengenlehre, Leipzig, 1914 (reprinted, New York, 1949).

4. Bjarni Jónsson and Alfred Tarski, Boolean algebras with operators. Part I, Amer. J. Math. 73 (1951), 891 - 939.

5. Andrzej Mostowski and Alfred Tarski, Boolesche Ringe mit geordneter Basis, Fund. Math. 32 (1939), 69-86.

6. Alfred Tarski, A lattice-theoretical fixpoint theorem and its applications, Pacific J. Math. 5 (1955), 285 - 309. 



\section{PACIFIC JOURNAL OF MATHEMATICS}

\section{EDITORS}

\author{
H. L. ROYDEN \\ Stanford University \\ Stanford, California \\ E. Hewitt \\ University of Washington \\ Seattle 5 , Washington
}

R. P. Dilworth

California Institute of Technology Pasadena 4, California

A. HorN*

University of California

Los Angeles 24, California

\section{ASSOCIATE EDITORS}

\author{
H. BUSEMANN \\ HERBERT FEDERER \\ MARSHALL HALL
}

\author{
P. R. HALMOS \\ HEINZ HOPF
}

ALFRED HORN
R. D. JAMES

BORGE JESSEN

PAUL LÉVY
GEORGE PÓLYA

J. J. STOKER

KOSAKU YOSIDA

\section{SPONSORS}

UNIVERSITY OF BRITISH COLUMBIA

CALIFORNIA INSTITUTE OF TECHNOLOGY

UNIVERSITY OF CALIFORNIA, BERKELEY

UNIVERSITY OF CALIFORNIA, DAVIS

UNIVERSITY OF CALIFORNIA, LOS ANGELES

UNIVERSITY OF CALIFORNIA, SANTA BARBARA

MONTANA STATE UNIVERSITY

UNIVERSITY OF NEVADA

OREGON STATE COLLEGE

UNIVERSITY OF OREGON

UNIVERSITY OF SOUTHERN CALIFORNIA

\author{
STANFORD RESEARCH INSTITUTE \\ STANFORD UNIVERSITY \\ UNIVERSITY OF UTAH \\ WASHINGTON STATE COLLEGE \\ UNIVERSITY OF WASHINGTON
}

AMERICAN MATHEMATICAL SOCIETY HUGHES AIRCRAFT COMPANY SHELL DEVELOPMENT COMPANY

Mathematical papers intended for publication in the Pacific Journal of Mathematics should be typewritten (double spaced), and the author should keep a complete copy. Manuscripts may be sent to any of the editors. Manuscripts intended for the outgoing editors should be sent to their successors. All other communications to the editors should be addressed to the managing editor, Alfred Horn at the University of California, Los Angeles 24, California.

50 reprints of each article are furnished free of charge; additional copies may be obtained at cost in multiples of 50 .

The Pacific Journal of Mathematics is published quarterly, in March, June, September, and December. The price per volume (4 numbers) is $\$ 12.00$; single issues, $\$ 3.50$. Back numbers are available. Special price to individual faculty members of supporting institutions and to individual members of the American Mathematical Society: $\$ 4.00$ per volume; single issues, $\$ 1.25$.

Subscriptions, orders for back numbers, and changes of address should be sent to Pacific Journal of Mathematics, c/o University of California Press, Berkeley 4, California.

Printed at Kokusai Bunken Insatsusha (International Academic Printing Co., Ltd.), No. 10, 1-chome, Fujimi-cho, Chiyoda-ku, Tokyo, Japan.

* During the absence of E. G. Straus.

PUBLISHED BY PACIFIC JOURNAL OF MATHEMATICS, A NON-PROFIT CORPORATION COPYRIGHT 1955 BY PACIFIC JOURNAL OF MATHEMATICS 


\section{Pacific Journal of Mathematics}

\section{Vol. 5, No. $2 \quad$ October, 1955}

Leonard M. Blumenthal, An extension of a theorem of Jordan and von

Neumann ........................................ 161

L. Carlitz, Note on the multiplication formulas for the Jacobi elliptic functions.......................................... 169

L. Carlitz, The number of solutions of certain types of equations in a finite

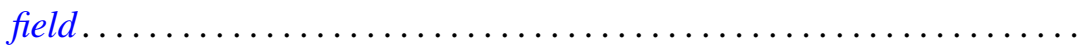

George Bernard Dantzig, Alexander Orden and Philip Wolfe, The generalized simplex method for minimizing a linear form under linear

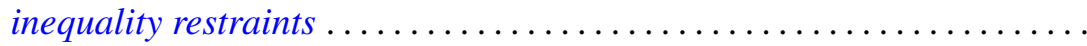

Arthur Pentland Dempster and Seymour Schuster, Constructions for poles and polars in n-dimensions . . . . . . . . . . . . . . . . . . . . 197

Franklin Haimo, Power-type endomorphisms of some class 2 groups ...... 201

Lloyd Kenneth Jackson, On generalized subharmonic functions ......... 215

Samuel Karlin, On the renewal equation ...................... 229

Frank R. Olson, Some determinants involving Bernoulli and Euler numbers of higher order................................ 259

R. S. Phillips, The adjoint semi-group ........................ 269

Alfred Tarski, A lattice-theoretical fixpoint theorem and its applications ... 285

Anne C. Davis, A characterization of complete lattices .............. 311 